\title{
ON THE ESSENTIAL SPECTRUM OF THE SUM OF SELF-ADJOINT OPERATORS AND THE CLOSEDNESS OF THE SUM OF OPERATOR RANGES
}

\author{
IVAN S. FESHCHENKO
}

\begin{abstract}
Let $\mathcal{H}$ be a complex Hilbert space, and $A_{1}, \ldots, A_{N}$ be bounded self-adjoint operators in $\mathcal{H}$ such that $A_{i} A_{j}$ is compact for any $i \neq j$. It is well-known that $\sigma_{e}\left(\sum_{i=1}^{N} A_{i}\right) \backslash\{0\}=\left(\cup_{i=1}^{N} \sigma_{e}\left(A_{i}\right)\right) \backslash\{0\}$, where $\sigma_{e}(B)$ stands for the essential spectrum of a bounded self-adjoint operator $B$.

In this paper we get necessary and sufficient conditions for $0 \in \sigma_{e}\left(\sum_{i=1}^{N} A_{i}\right)$. This conditions are formulated in terms of the projection valued spectral measures of $A_{i}, i=1, \ldots, N$. Using this result, we obtain necessary and sufficient conditions for the sum of ranges of $A_{i}, i=1, \ldots, N$ to be closed.
\end{abstract}

\section{INTRODUCTION}

Let $\mathcal{H}$ be a complex Hilbert space, and $A_{1}, \ldots, A_{N}$ be $N \geqslant 2$ bounded selfadjoint operators in $\mathcal{H}$ such that $A_{i} A_{j}$ is compact for $i \neq j$. Define

$$
A=\sum_{i=1}^{N} A_{i} .
$$

1.1. On the essential spectrum of the sum of self-adjoint operators. The following result on the essential spectrum of $A, \sigma_{e}(A)$, is well-known and has applications in scattering theory and spectral analysis of Hankel operators (see, e.g., [7, Proposition 3.1],[6, Chapter 10, Lemma 1.5]).

Theorem A. We have

$$
\sigma_{e}(A) \backslash\{0\}=\left(\bigcup_{i=1}^{N} \sigma_{e}\left(A_{i}\right)\right) \backslash\{0\} .
$$

For convenience of the reader, we will prove Theorem A (following [7]) in Section 3.

What one can say about the point 0 ?

If $\mathcal{H}$ is infinite dimensional, then $0 \in \cup_{i=1}^{N} \sigma_{e}\left(A_{i}\right)$. Indeed, it is easily checked, that if $B_{1}, B_{2}$ are bounded self-adjoint operators in $\mathcal{H}$ such that $B_{1} B_{2}$ is compact, then $0 \notin \sigma_{e}\left(B_{1}\right) \Rightarrow B_{2}$ is compact. Hence, if $0 \notin \sigma_{e}\left(A_{1}\right)$, then $A_{2}$ is compact, and, consequently, $\sigma_{e}\left(A_{2}\right)=\{0\}$.

Date: Received: 26 December 2012; Accepted: 14 April 2013.

2010 Mathematics Subject Classification. Primary 47B15; Secondary 46C07.

Key words and phrases. Self-adjoint operator, compact operator, essential spectrum, sum of operator ranges, closedness. 
Thus, the following question arises naturally: when $0 \in \sigma_{e}(A)$ ? We will give an answer to this question in terms of the projection valued spectral measures of $A_{i}, E_{A_{i}}(\cdot), i=1, \ldots, N$. Define the subspace

$$
\mathcal{H}_{\varepsilon}=\mathcal{H}_{\varepsilon}\left(A_{1}, \ldots, A_{N}\right)=\bigcap_{i=1}^{N} E_{A_{i}}([-\varepsilon, \varepsilon]) \mathcal{H}, \quad \varepsilon \geqslant 0 .
$$

Main Theorem. $0 \in \sigma_{e}(A)$ if and only if the subspace $\mathcal{H}_{\varepsilon}$ is infinite dimensional for any $\varepsilon>0$.

1.2. On the closedness of the sum of operator ranges. The problem on the closedness of the sum of operator ranges (criteria for the sum of operator ranges to be closed, properties of collections of operators $X_{i}$ such that $\sum_{i=1}^{n} \operatorname{Ran}\left(X_{i}\right)$ is closed), in particular, the problem on the closedness of the sum of $n$ subspaces of a Hilbert space (criteria for the sum of $n$ subspaces of a Hilbert space to be closed, properties of collections of subspaces with closed sum), is an important problem of functional analysis. This problem was studied in numerous publications and has many applications in various branches of mathematics, see, for example, [4] and the bibliography therein (unfortunately, English translation of the Russian original of this paper in some places is bad. For example, the word "closeness" must be replaced by "closedness"), [3], [1].

We consider the following question: when the sum of ranges of $A_{i}$,

$$
\sum_{i=1}^{N} \operatorname{Ran}\left(A_{i}\right)=\left\{\sum_{i=1}^{N} y_{i} \mid y_{i} \in \operatorname{Ran}\left(A_{i}\right)\right\}=\left\{\sum_{i=1}^{N} A_{i} x_{i} \mid x_{i} \in \mathcal{H}\right\},
$$

is closed? This question arises naturally in connection with the following result having applications in theoretical tomography (see [5] and the bibliography therein).

Theorem B. Let $P_{1}, \ldots, P_{N}$ be orthogonal projections in $\mathcal{H}$. Suppose $P_{i} P_{j}$ is compact for $i \neq j$. Then $\sum_{i=1}^{N} \operatorname{Ran}\left(P_{i}\right)$ is closed.

Remark 1. Theorem B is stated only for Hilbert spaces, while it was proved by Lars Svensson for reflexive Banach spaces [8], by Harald Lang for Frechet spaces [5], and by Lars Svensson for Hausdorff locally compact topological vector spaces [9].

Using Main Theorem, we prove a criterion for $\sum_{i=1}^{N} \operatorname{Ran}\left(A_{i}\right)$ to be closed. Recall that the subspaces $\mathcal{H}_{\varepsilon}, \varepsilon \geqslant 0$, are defined by (1.1). Clearly,

$$
\mathcal{H}_{0}=\bigcap_{i=1}^{N} \operatorname{Ker}\left(A_{i}\right)
$$

Note that $\mathcal{H}_{0} \subset \mathcal{H}_{\varepsilon}$ for any $\varepsilon>0$.

Theorem 1. $\sum_{i=1}^{N} \operatorname{Ran}\left(A_{i}\right)$ is closed if and only if $\mathcal{H}_{\varepsilon}=\mathcal{H}_{0}$ for some $\varepsilon>0$.

Remark 2. We will prove that if $\mathcal{H}_{\varepsilon} \ominus \mathcal{H}_{0}$ is finite dimensional for some $\varepsilon>0$, then $\sum_{i=1}^{N} \operatorname{Ran}\left(A_{i}\right)$ is closed. 
Remark 3. If $\sum_{i=1}^{N} \operatorname{Ran}\left(A_{i}\right)$ is closed, then $\sum_{i=1}^{N} \operatorname{Ran}\left(A_{i}\right)=\mathcal{H} \ominus \mathcal{H}_{0}$. Indeed, suppose $\sum_{i=1}^{N} \operatorname{Ran}\left(A_{i}\right)$ is closed. We have

$$
\mathcal{H} \ominus\left(\sum_{i=1}^{N} \operatorname{Ran}\left(A_{i}\right)\right)=\bigcap_{i=1}^{N}\left(\mathcal{H} \ominus \operatorname{Ran}\left(A_{i}\right)\right)=\bigcap_{i=1}^{N} \operatorname{Ker}\left(A_{i}\right)=\mathcal{H}_{0} .
$$

Hence, $\sum_{i=1}^{N} \operatorname{Ran}\left(A_{i}\right)=\mathcal{H} \ominus \mathcal{H}_{0}$.

Using this criterion for $\sum_{i=1}^{N} \operatorname{Ran}\left(A_{i}\right)$ to be closed, we get the following generalization of Theorem B.

Theorem 2. Let $A_{p, i}: \mathcal{K}_{p, i} \rightarrow \mathcal{H}, i=1, \ldots, N_{p}, p=1, \ldots, m$ be bounded linear operators (here $\mathcal{K}_{p, i}$ are Hilbert spaces, $m, N_{1}, \ldots, N_{m}$ are natural numbers) such that $A_{p, i}^{*} A_{q, j}$ is compact for any $p \neq q, i=1, \ldots, N_{p}, j=1, \ldots, N_{q}$. If $\mathcal{R}_{p}=\sum_{i=1}^{N_{p}} \operatorname{Ran}\left(A_{p, i}\right)$ is closed for any $p=1, \ldots, m$, then $\sum_{p=1}^{m} \mathcal{R}_{p}$ is closed.

Corollary 1. If Ran $\left(A_{i}\right)$ is closed for $i=1, \ldots, N$, then $\sum_{i=1}^{N} \operatorname{Ran}\left(A_{i}\right)$ is closed.

Note that our proof of Theorem 2 is not a generalization of the proof of Theorem B (and we don't use Theorem B in the proof of Theorem 2).

1.3. Notation. In this paper we consider only complex Hilbert spaces usually denoted by the letters $\mathcal{H}, \mathcal{K}$. The scalar product in $\mathcal{H}$ is denoted by $\langle\cdot, \cdot \cdot$, and $\|\cdot\|$ stands for the corresponding norm, $\|x\|^{2}=\langle x, x\rangle$. The identity operator on $\mathcal{H}$ is denoted by $I_{\mathcal{H}}$ or simply $I$ if it is clear which Hilbert space is being considered. For a bounded linear operator $X: \mathcal{H} \rightarrow \mathcal{H}, \sigma(X)$ denotes the spectrum of the operator $X$.

\section{Auxiliary Results and notions}

2.1. The essential spectrum of a self-adjoint operator. Following [2, Chapter 9], we recall the definition and some properties of the essential spectrum of a self-adjoint operator.

Let $A$ be a bounded self-adjoint operator in a complex Hilbert space $\mathcal{H}$. The essential spectrum of $A, \sigma_{e}(A)$, is the set of all $\lambda \in \sigma(A)$ such that either $\lambda$ is a limit point of $\sigma(A)$ or $\operatorname{Ker}(A-\lambda I)$ is infinite-dimensional. The set $\sigma_{d}(A)=$ $\sigma(A) \backslash \sigma_{e}(A)$ is called the discrete spectrum of $A$. Clearly, $\lambda \in \sigma_{d}(A)$ if and only if $\lambda$ is an isolated point of $\sigma(A)$ and $\operatorname{Ker}(A-\lambda I)$ is finite-dimensional.

It terms of the spectral measure $E_{A}(\cdot)$ the essential spectrum of $A$ can be characterized as follows: $\lambda \in \sigma_{e}(A)$ if and only if $E_{A}((\lambda-\varepsilon, \lambda+\varepsilon)) \mathcal{H}$ is infinitedimensional for any $\varepsilon>0$.

More convenient for us is a description of $\sigma_{e}(A)$ in terms of singular sequences. Recall that a sequence $\left\{x_{k} \mid k \geqslant 1\right\}, x_{k} \in \mathcal{H}, k \geqslant 1$, is called a singular sequence for $A$ at the point $\lambda$ if

(1) $x_{k} \rightarrow 0$ weakly as $k \rightarrow \infty$;

(2) $x_{k}$ does not converge to 0 as $k \rightarrow \infty$;

(3) $(A-\lambda I) x_{k} \rightarrow 0$ as $k \rightarrow \infty$.

Let us denote by $\operatorname{sing}(A, \lambda)$ the set of all sequences $\left\{x_{k} \mid k \geqslant 1\right\}$ which are singular for $A$ at $\lambda$. 
Proposition 2.1. $\lambda \in \sigma_{e}(A)$ if and only if $\operatorname{sing}(A, \lambda) \neq \varnothing$.

\subsection{On the sum of operator ranges.}

Proposition 2.2. Let $\mathcal{K}_{1}, \ldots, \mathcal{K}_{n}$ and $\mathcal{H}$ be Hilbert spaces, $B_{i}: \mathcal{K}_{i} \rightarrow \mathcal{H}$ a bounded linear operators, $i=1, \ldots, n$. If $\sum_{i=1}^{n} \operatorname{Ran}\left(B_{i}\right)=\mathcal{H}$, then $\sum_{i=1}^{n} B_{i} B_{i}^{*} \geqslant$ $\varepsilon I$ for some $\varepsilon>0$.

Proof. Define an operator $B: \mathcal{K}_{1} \oplus \ldots \oplus \mathcal{K}_{n} \rightarrow \mathcal{H}$ by

$$
B\left(x_{1}, \ldots, x_{n}\right)=\sum_{i=1}^{n} B_{i} x_{i}, \quad x_{i} \in \mathcal{K}_{i}, \quad i=1, \ldots, n .
$$

Then $B^{*}: \mathcal{H} \rightarrow \mathcal{K}_{1} \oplus \ldots \oplus \mathcal{K}_{n}$ and

$$
B^{*} x=\left(B_{1}^{*} x, \ldots, B_{n}^{*} x\right), \quad x \in \mathcal{H} .
$$

Since $\operatorname{Ran}(B)=\sum_{i=1}^{n} \operatorname{Ran}\left(B_{i}\right)=\mathcal{H}$, we conclude that $B^{*}$ is an isomorphic embedding, that is, there exists $c>0$ such that $\left\|B^{*} x\right\| \geqslant c\|x\|, x \in \mathcal{H}$. We have

$$
\left\|B^{*} x\right\|^{2}=\sum_{i=1}^{n}\left\|B_{i}^{*} x\right\|^{2}=\sum_{i=1}^{n}\left\langle B_{i} B_{i}^{*} x, x\right\rangle=\left\langle\left(\sum_{i=1}^{n} B_{i} B_{i}^{*}\right) x, x\right\rangle .
$$

Hence, $\sum_{i=1}^{n} B_{i} B_{i}^{*} \geqslant c^{2} I$.

We will need a simple corollary of Proposition 2.2.

Corollary 2. Let $\mathcal{K}_{1}, \ldots, \mathcal{K}_{n}$ and $\mathcal{H}$ be Hilbert spaces, $B_{i}: \mathcal{K}_{i} \rightarrow \mathcal{H}$ a bounded linear operators, $i=1, \ldots, n$. If $\sum_{i=1}^{n} \operatorname{Ran}\left(B_{i}\right)$ is closed, then

$$
\sum_{i=1}^{n} \operatorname{Ran}\left(B_{i}\right)=\operatorname{Ran}\left(\sum_{i=1}^{n} B_{i} B_{i}^{*}\right) .
$$

The following proposition is well-known.

Proposition 2.3. Let $B$ be a bounded self-adjoint operator in $\mathcal{H}$. $\operatorname{Ran}(B)$ is closed if and only if $\sigma(B) \cap((-\varepsilon, 0) \cup(0, \varepsilon))=\varnothing$ for some $\varepsilon>0$.

The proof is trivial and is omitted.

\section{Proof of Theorem A}

To prove Theorem A, we need the following simple lemma which shows relation between singular sequences of two bounded self-adjoint operators whose product is compact.

Lemma 3.1. Let $B_{1}, B_{2}$ be bounded self-adjoint operators in $\mathcal{H}$. Suppose $B_{1} B_{2}$ is compact. If $\left\{x_{k} \mid k \geqslant 1\right\} \in \operatorname{sing}\left(B_{2}, \lambda\right)$, where $\lambda \neq 0$, then $\left\{x_{k} \mid k \geqslant 1\right\} \in$ $\operatorname{sing}\left(B_{1}, 0\right)$.

Proof. We have $\left(B_{2}-\lambda I\right) x_{k} \rightarrow 0, k \rightarrow \infty$. It follows that $B_{1}\left(B_{2}-\lambda I\right) x_{k} \rightarrow 0$, $k \rightarrow \infty$, that is, $B_{1} B_{2} x_{k}-\lambda B_{1} x_{k} \rightarrow 0, k \rightarrow \infty$. Since $B_{1} B_{2}$ is compact and $x_{k} \rightarrow 0$ weakly, we conclude that $B_{1} B_{2} x_{k} \rightarrow 0$. Hence, $\lambda B_{1} x_{k} \rightarrow 0, k \rightarrow \infty$, whence, $B_{1} x_{k} \rightarrow 0, k \rightarrow \infty$. Therefore, $\left\{x_{k} \mid k \geqslant 1\right\} \in \operatorname{sing}\left(B_{1}, 0\right)$. 
Proof of Theorem A. 1. Suppose that $\lambda \in \sigma_{e}\left(A_{i}\right)$ for some $i$, and $\lambda \neq 0$. Let us show that $\lambda \in \sigma_{e}(A)$. There exists a sequence $\left\{x_{k} \mid k \geqslant 1\right\} \in \operatorname{sing}\left(A_{i}, \lambda\right)$. By Lemma 3.1, $\left\{x_{k} \mid k \geqslant 1\right\} \in \operatorname{sing}\left(A_{j}, 0\right)$ for $j \neq i$. We have

$$
(A-\lambda I) x_{k}=\left(A_{i}-\lambda I\right) x_{k}+\sum_{j \neq i} A_{j} x_{k} \rightarrow 0, \quad k \rightarrow \infty .
$$

Hence, $\left\{x_{k} \mid k \geqslant 1\right\} \in \operatorname{sing}(A, \lambda)$, whence $\lambda \in \sigma_{e}(A)$.

2. Suppose that $\lambda \in \sigma_{e}(A), \lambda \neq 0$. Let us prove that $\lambda \in \sigma_{e}\left(A_{i}\right)$ for some $i$. There exists $\left\{x_{k} \mid k \geqslant 1\right\} \in \operatorname{sing}(A, \lambda)$. We have

$$
(A-\lambda I) x_{k}=\left(\sum_{j=1}^{N} A_{j}-\lambda I\right) x_{k} \rightarrow 0, \quad k \rightarrow \infty .
$$

Let $i \in\{1, \ldots, n\}$. Then $A_{i}\left(\sum_{j=1}^{N} A_{j}-\lambda I\right) x_{k} \rightarrow 0, k \rightarrow \infty$, that is, $\sum_{j=1}^{N} A_{i} A_{j} x_{k}-$ $\lambda A_{i} x_{k} \rightarrow 0, k \rightarrow \infty$. Since $A_{i} A_{j}$ is compact for $j \neq i$ and $x_{k} \rightarrow 0$ weakly, we conclude that $A_{i} A_{j} x_{k} \rightarrow 0, k \rightarrow \infty$ for $j \neq i$. Hence, $A_{i}^{2} x_{k}-\lambda A_{i} x_{k} \rightarrow 0, k \rightarrow \infty$, i.e., $\left(A_{i}-\lambda I\right) A_{i} x_{k} \rightarrow 0, k \rightarrow \infty$.

Suppose that there exists $i$ such that the sequence $A_{i} x_{k}$ does not converge to 0 as $k \rightarrow \infty$. Then $\left\{A_{i} x_{k} \mid k \geqslant 1\right\} \in \operatorname{sing}\left(A_{i}, \lambda\right)$, whence $\lambda \in \sigma_{e}\left(A_{i}\right)$.

Now assume that $A_{i} x_{k} \rightarrow 0, k \rightarrow \infty$ for any $i=1, \ldots, N$. From (3.1) it follows that $\lambda x_{k} \rightarrow 0, k \rightarrow \infty$. Hence, $x_{k} \rightarrow 0, k \rightarrow \infty$, a contradiction.

The proof is complete.

\section{Proof of Main Theorem}

To prove Main Theorem, we need the following two lemmas.

Lemma 4.1. Let $B, C$ be bounded self-adjoint operators in $\mathcal{H}$. If $B C$ is compact, then

$$
E_{B}(\mathbb{R} \backslash[-\varepsilon, \varepsilon]) E_{C}(\mathbb{R} \backslash[-\delta, \delta])
$$

is compact for any $\varepsilon, \delta>0$.

Proof. Set $P=E_{B}(\mathbb{R} \backslash[-\varepsilon, \varepsilon]), Q=E_{C}(\mathbb{R} \backslash[-\delta, \delta])$. Then $B^{2} \geqslant \varepsilon^{2} P, C^{2} \geqslant \delta^{2} Q$.

Since $B C$ is compact, we see that $B C^{2} B$ is compact. Clearly, $B C^{2} B \geqslant \delta^{2} B Q B$. Hence, $B Q B$ is compact. But $B Q B=(B Q)(B Q)^{*}$. Consequently, $B Q$ is compact. Then $Q B^{2} Q$ is compact. Clearly, $Q B^{2} Q \geqslant \varepsilon^{2} Q P Q$. Hence, $Q P Q$ is compact. But $Q P Q=(Q P)(Q P)^{*}$. Consequently, $Q P$ is compact. It follows that $P Q=(Q P)^{*}$ is compact.

Lemma 4.2. Let $P_{1}, \ldots, P_{n}$ be orthogonal projections in $\mathcal{H}$. Suppose that $P_{i} P_{j}$ is compact for any $i \neq j$. Then there exists $\varepsilon>0$ such that

$$
\sigma\left(\sum_{i=1}^{n} P_{i}\right) \cap(0, \varepsilon)=\varnothing .
$$

Proof. Set $\mathcal{K}_{i}=\operatorname{Ran}\left(P_{i}\right), i=1, \ldots, n$. Define an operator $\Gamma: \mathcal{H} \rightarrow \mathcal{K}_{1} \oplus \ldots \oplus \mathcal{K}_{n}$ by

$$
\Gamma x=\left(P_{1} x, \ldots, P_{n} x\right), \quad x \in \mathcal{H} .
$$


Then $\Gamma^{*}: \mathcal{K}_{1} \oplus \ldots \oplus \mathcal{K}_{n} \rightarrow \mathcal{H}$ and

$$
\Gamma^{*}\left(x_{1}, \ldots, x_{n}\right)=x_{1}+\ldots+x_{n}, \quad x_{i} \in \mathcal{K}_{i}, \quad i=1, \ldots, n .
$$

Hence, $\Gamma^{*} \Gamma=\sum_{i=1}^{n} P_{i}$. Clearly, the operator $\Gamma \Gamma^{*}: \mathcal{K}_{1} \oplus \ldots \oplus \mathcal{K}_{n} \rightarrow \mathcal{K}_{1} \oplus \ldots \oplus \mathcal{K}_{n}$ and its block decomposition is equal to

$$
\Gamma \Gamma^{*}=\left(P_{i}\left\lceil\mathcal{K}_{j}: \mathcal{K}_{j} \rightarrow \mathcal{K}_{i} \mid 1 \leqslant i, j \leqslant n\right)\right.
$$

Since $P_{i} P_{j}$ is compact for any $i \neq j$, we conclude that

$$
\left(\Gamma \Gamma^{*}\right)_{i, j}=P_{i}\left\lceil\mathcal{K}_{j}=P_{i} P_{j}\left\lceil\mathcal{K}_{j}\right.\right.
$$

is compact for $i \neq j$. Consequently, $\Gamma \Gamma^{*}-I$ is compact. By the Weyl theorem, $\sigma_{e}\left(\Gamma \Gamma^{*}\right)=\sigma_{e}(I) \subset\{1\}$. Hence, $0 \notin \sigma_{e}\left(\Gamma \Gamma^{*}\right)$, whence $\sigma\left(\Gamma \Gamma^{*}\right) \cap(0, \varepsilon)=\varnothing$ for some $\varepsilon>0$. Since $\sigma\left(\Gamma^{*} \Gamma\right) \backslash\{0\}=\sigma\left(\Gamma \Gamma^{*}\right) \backslash\{0\}$, we conclude that $\sigma\left(\sum_{i=1}^{n} P_{i}\right) \cap(0, \varepsilon)=$ $\varnothing$.

Proof of Main Theorem. 1. Suppose the subspace $\mathcal{H}_{\varepsilon}$ is infinite dimensional for any $\varepsilon>0$. We claim that $0 \in \sigma_{e}(A)$. To prove this, we construct $\left\{x_{k} \mid k \geqslant 1\right\} \in$ $\operatorname{sing}(A, 0)$ as follows. Take

$$
x_{1} \in \mathcal{H}_{1}, \quad\left\|x_{1}\right\|=1 .
$$

Suppose $x_{1}, \ldots, x_{k}$ have already been defined. Clearly, we can choose

$$
x_{k+1} \in \mathcal{H}_{1 /(k+1)}, \quad\left\|x_{k+1}\right\|=1
$$

such that $x_{k+1}$ is orthogonal to $x_{i}, i=1, \ldots, k$. Hence, we obtain the sequence $\left\{x_{k} \mid k \geqslant 1\right\}$. By the construction, $\left\{x_{k} \mid k \geqslant 1\right\}$ is orthonormal. Moreover, $\left\|A_{i} x_{k}\right\| \leqslant 1 / k, i=1, \ldots, N$. It follows that $\left\|A x_{k}\right\| \leqslant N / k, k \geqslant 1$. Consequently, $A x_{k} \rightarrow 0$ as $k \rightarrow \infty$. Hence, $\left\{x_{k} \mid k \geqslant 1\right\} \in \operatorname{sing}(A, 0)$, whence $0 \in \sigma_{e}(A)$.

2. Suppose $\mathcal{H}_{\varepsilon}$ is finite dimensional for some $\varepsilon>0$. Let us show that $0 \notin \sigma_{e}(A)$. Define $P_{i}=E_{A_{i}}(\mathbb{R} \backslash[-\varepsilon, \varepsilon]), i=1, \ldots, N$. By Lemma 4.1, $P_{i} P_{j}$ is compact for any $i \neq j$. By Lemma 4.2, there exists $\delta>0$ such that $\sigma\left(\sum_{i=1}^{N} P_{i}\right) \cap(0, \delta)=\varnothing$. It follows that

$$
\sum_{i=1}^{N} P_{i}+\delta Q \geqslant \delta I
$$

where $Q$ is the orthogonal projection onto $\operatorname{Ker}\left(\sum_{i=1}^{N} P_{i}\right)$. Clearly,

$$
\operatorname{Ker}\left(\sum_{i=1}^{N} P_{i}\right)=\bigcap_{i=1}^{N} \operatorname{Ker}\left(P_{i}\right)=\bigcap_{i=1}^{N} E_{A_{i}}([-\varepsilon, \varepsilon]) \mathcal{H}=\mathcal{H}_{\varepsilon} .
$$

Hence, $Q$ is a finite dimensional orthogonal projection. Since $A_{i}^{2} \geqslant \varepsilon^{2} P_{i}, i=$ $1, \ldots, N$, we have

$$
\sum_{i=1}^{N} A_{i}^{2}+\varepsilon^{2} \delta Q \geqslant \sum_{i=1}^{N} \varepsilon^{2} P_{i}+\varepsilon^{2} \delta Q \geqslant \varepsilon^{2} \delta I .
$$

Hence,

$$
\sum_{i=1}^{N} A_{i}^{2}+\mu Q \geqslant \mu I
$$


where $\mu=\varepsilon^{2} \delta$.

Now we are in a position to prove that $0 \notin \sigma_{e}(A)$. Suppose that $0 \in \sigma_{e}(A)$. There exists a sequence $\left\{x_{k} \mid k \geqslant 1\right\} \in \operatorname{sing}(A, 0)$. We have $\left(\sum_{j=1}^{N} A_{j}\right) x_{k} \rightarrow 0$ as $k \rightarrow \infty$. Let $i \in\{1, \ldots, N\}$. Then $A_{i}\left(\sum_{j=1}^{N} A_{j}\right) x_{k} \rightarrow 0$ as $k \rightarrow \infty$, that is, $\sum_{j=1}^{N} A_{i} A_{j} x_{k} \rightarrow 0$ as $k \rightarrow \infty$. Since $A_{i} A_{j}$ is compact for $j \neq i$ and $x_{k} \rightarrow 0$ weakly, we conclude that $A_{i} A_{j} x_{k} \rightarrow 0$ as $k \rightarrow \infty$ for $j \neq i$. Consequently, $A_{i}^{2} x_{k} \rightarrow 0$, $k \rightarrow \infty$. Since $\left\{x_{k} \mid k \geqslant 1\right\}$ is bounded, we conclude that $\left\langle A_{i}^{2} x_{k}, x_{k}\right\rangle \rightarrow 0, k \rightarrow \infty$. Hence, $\left\|A_{i} x_{k}\right\|^{2} \rightarrow 0, k \rightarrow \infty$, whence $A_{i} x_{k} \rightarrow 0, k \rightarrow \infty$. Since $Q$ is a finite dimensional operator and $x_{k} \rightarrow 0$ weakly, we conclude that $Q x_{k} \rightarrow 0, k \rightarrow \infty$. From (4.1) it follows that

$$
\sum_{i=1}^{N}\left\langle A_{i}^{2} x_{k}, x_{k}\right\rangle+\mu\left\langle Q x_{k}, x_{k}\right\rangle \geqslant \mu\left\|x_{k}\right\|^{2},
$$

that is,

$$
\sum_{i=1}^{N}\left\|A_{i} x_{k}\right\|^{2}+\mu\left\|Q x_{k}\right\|^{2} \geqslant \mu\left\|x_{k}\right\|^{2}
$$

It follows that $x_{k} \rightarrow 0$ as $k \rightarrow \infty$, a contradiction.

Hence, $0 \notin \sigma_{e}(A)$.

\section{Proof of TheOrem 1}

Proof of Theorem 1. Let $\mathcal{K}=\mathcal{H} \ominus \mathcal{H}_{0}$, then $\mathcal{H}=\mathcal{H}_{0} \oplus \mathcal{K}$. With respect to this orthogonal decomposition $A_{i}=0 \oplus B_{i}, i=1, \ldots, N$, where $B_{i}$ is a bounded selfadjoint operator in $\mathcal{K}$. Since $\mathcal{H}_{0}=\cap_{i=1}^{N} \operatorname{Ker}\left(A_{i}\right)$, we see that $\cap_{i=1}^{N} \operatorname{Ker}\left(B_{i}\right)=\{0\}$. Since $A_{i} A_{j}$ is compact for $i \neq j$, we conclude that $B_{i} B_{j}$ is compact for $i \neq j$.

1. Suppose $\sum_{i=1}^{N} \operatorname{Ran}\left(A_{i}\right)$ is closed. Let us show that $\mathcal{H}_{\delta}=\mathcal{H}_{0}$ for some $\delta>0$. $\sum_{i=1}^{N} \operatorname{Ran}\left(B_{i}\right)=\sum_{i=1}^{N} \operatorname{Ran}\left(A_{i}\right)$ is closed. Since

$$
\mathcal{K} \ominus\left(\sum_{i=1}^{N} \operatorname{Ran}\left(B_{i}\right)\right)=\bigcap_{i=1}^{N}\left(\mathcal{K} \ominus \operatorname{Ran}\left(B_{i}\right)\right)=\bigcap_{i=1}^{N} \operatorname{Ker}\left(B_{i}\right)=\{0\},
$$

we conclude that $\sum_{i=1}^{N} \operatorname{Ran}\left(B_{i}\right)=\mathcal{K}$. By Proposition 2.2, $\sum_{i=1}^{N} B_{i}^{2} \geqslant \varepsilon I$ for some $\varepsilon>0$. Set $\delta=\sqrt{\varepsilon /(2 N)}$. We claim that $\mathcal{H}_{\delta}=\mathcal{H}_{0}$. Let us prove this. We have $E_{A_{i}}(\cdot)=E_{0}(\cdot) \oplus E_{B_{i}}(\cdot)$. Hence, $E_{A_{i}}([-\delta, \delta])=I \oplus E_{B_{i}}([-\delta, \delta])$, whence $E_{A_{i}}([-\delta, \delta]) \mathcal{H}=\mathcal{H}_{0} \oplus E_{B_{i}}([-\delta, \delta]) \mathcal{K}$. Thus

$$
\mathcal{H}_{\delta}=\mathcal{H}_{0} \oplus \cap_{i=1}^{N} E_{B_{i}}([-\delta, \delta]) \mathcal{K} .
$$

But $\cap_{i=1}^{N} E_{B_{i}}([-\delta, \delta]) \mathcal{K}=\{0\}$. Indeed, suppose $x \in \cap_{i=1}^{N} E_{B_{i}}([-\delta, \delta]) \mathcal{K}$ and $x \neq 0$. Then $\left\|B_{i} x\right\| \leqslant \delta\|x\|, i=1, \ldots, N$. Hence,

$$
\left\langle\left(\sum_{i=1}^{N} B_{i}^{2}\right) x, x\right\rangle=\sum_{i=1}^{N}\left\|B_{i} x\right\|^{2} \leqslant N \delta^{2}\|x\|^{2}=\frac{\varepsilon}{2}\|x\|^{2} .
$$

But $\left\langle\left(\sum_{i=1}^{N} B_{i}^{2}\right) x, x\right\rangle \geqslant \varepsilon\|x\|^{2}$. We get a contradiction. Hence, $\cap_{i=1}^{N} E_{B_{i}}([-\delta, \delta]) \mathcal{K}=$ $\{0\}$, and, consequently, $\mathcal{H}_{\delta}=\mathcal{H}_{0}$. 
2. Let us prove that if $\mathcal{H}_{\varepsilon}=\mathcal{H}_{0}$ for some $\varepsilon>0$, then $\sum_{i=1}^{N} \operatorname{Ran}\left(A_{i}\right)$ is closed. We will prove a more stronger fact: if $\mathcal{H}_{\varepsilon} \ominus \mathcal{H}_{0}$ is finite dimensional for some $\varepsilon>0$, then $\sum_{i=1}^{N} \operatorname{Ran}\left(A_{i}\right)$ is closed.

Since $A_{i}^{2}=0 \oplus B_{i}^{2}$, we see that $E_{A_{i}^{2}}(\cdot)=E_{0}(\cdot) \oplus E_{B_{i}^{2}}(\cdot)$. Hence, $E_{A_{i}^{2}}\left(\left[-\varepsilon^{2}, \varepsilon^{2}\right]\right)=$ $I \oplus E_{B_{i}^{2}}\left(\left[-\varepsilon^{2}, \varepsilon^{2}\right]\right)$. But $E_{A_{i}^{2}}\left(\left[-\varepsilon^{2}, \varepsilon^{2}\right]\right)=E_{A_{i}}([-\varepsilon, \varepsilon])$. Hence, $E_{A_{i}}([-\varepsilon, \varepsilon])=$ $I \oplus E_{B_{i}^{2}}\left(\left[-\varepsilon^{2}, \varepsilon^{2}\right]\right)$, whence $E_{A_{i}}([-\varepsilon, \varepsilon]) \mathcal{H}=\mathcal{H}_{0} \oplus E_{B_{i}^{2}}\left(\left[-\varepsilon^{2}, \varepsilon^{2}\right]\right) \mathcal{K}$. Thus

$$
\mathcal{H}_{\varepsilon}=\mathcal{H}_{0} \oplus \cap_{i=1}^{N} E_{B_{i}^{2}}\left(\left[-\varepsilon^{2}, \varepsilon^{2}\right]\right) \mathcal{K} .
$$

Thus, $\cap_{i=1}^{N} E_{B_{i}^{2}}\left(\left[-\varepsilon^{2}, \varepsilon^{2}\right]\right) \mathcal{K}$ is finite dimensional. By the Main Theorem, $0 \notin$ $\sigma_{e}\left(\sum_{i=1}^{N} B_{i}^{2}\right)$. Moreover, since

$$
\operatorname{Ker}\left(\sum_{i=1}^{N} B_{i}^{2}\right)=\bigcap_{i=1}^{N} \operatorname{Ker}\left(B_{i}\right)=\{0\}
$$

we conclude that $0 \notin \sigma_{d}\left(\sum_{i=1}^{N} B_{i}^{2}\right)$. Hence, $0 \notin \sigma\left(\sum_{i=1}^{N} B_{i}^{2}\right)$, that is, the operator $\sum_{i=1}^{N} B_{i}^{2}$ is invertible. It follows that $\sum_{i=1}^{N} \operatorname{Ran}\left(B_{i}\right)=\mathcal{K}$. Consequently, $\sum_{i=1}^{N} \operatorname{Ran}\left(A_{i}\right)=\mathcal{K}$ is closed.

\section{Proof of Theorem 2}

Proof of Theorem 2. Define

$$
B_{p}=\sum_{i=1}^{N_{p}} A_{p, i} A_{p, i}^{*}, \quad p=1, \ldots, m .
$$

Clearly, $B_{p}$ is a bounded self-adjoint operator in $\mathcal{H}$. Moreover, $B_{p} B_{q}$ is compact for $p \neq q$.

From Corollary 2 it follows that $\operatorname{Ran}\left(B_{p}\right)=\mathcal{R}_{p}, p=1, \ldots, m$. By Proposition 2.3, there exists $\varepsilon>0$ such that $\sigma\left(B_{p}\right) \cap((-\varepsilon, 0) \cup(0, \varepsilon))=\varnothing$ for $p=1, \ldots, m$. It follows that $E_{B_{p}}([-\varepsilon / 2, \varepsilon / 2])=E_{B_{p}}(\{0\})$, and, consequently, $E_{B_{p}}([-\varepsilon / 2, \varepsilon / 2]) \mathcal{H}=\operatorname{Ker}\left(B_{p}\right), p=1, \ldots, m$. Hence,

$$
\mathcal{H}_{\varepsilon / 2}\left(B_{1}, \ldots, B_{m}\right)=\bigcap_{p=1}^{m} E_{B_{p}}([-\varepsilon / 2, \varepsilon / 2]) \mathcal{H}=\bigcap_{p=1}^{m} \operatorname{Ker}\left(B_{p}\right)=\mathcal{H}_{0}\left(B_{1}, \ldots, B_{m}\right) .
$$

By Theorem 1, $\sum_{p=1}^{m} \operatorname{Ran}\left(B_{p}\right)=\sum_{p=1}^{m} \mathcal{R}_{p}$ is closed.

Acknowledgement. The author is grateful to Alexei Yu. Konstantinov for helpful suggestions.

\section{REFERENCES}

1. C. Badea, S. Grivaux, V. Muller, The rate of convergence in the method of alternating projections, St. Petersburg Math. J. 23 (2012), no. 3, 413-434.

2. M. S. Birman, M. Z. Solomjak, Spectral theory of self-adjoint operators in Hilbert space, D. Reidel Publishing Co., Dordrecht, 1987.

3. F. Deutsch, Best approximation in inner product spaces, CMS Books Math., SpringerVerlag, New York, 2001.

4. I. S. Feshchenko, On closeness of the sum of $n$ subspaces of a Hilbert space, Ukrainian Math. J. 63 (2012), no. 10, 1566-1622. 
5. H. Lang, On sums of subspaces in topological vector spaces and an application in theoretical tomography, Appl. Anal. 18 (1984), no. 4, 257-265.

6. V. V. Peller, Hankel operators and their applications, Springer Monogr. Math, 2002.

7. A. Pushnitski, D. Yafaev, A multichannel scheme in smooth scattering theory, J. Spectr. Theory, arXiv:1209.3238v1 [math.SP] (to appear).

8. L. Svensson, On the closure of sums of closed subspaces, Research report, Royal Inst. of Technology (Stockholm), 1981.

9. L. Svensson, Sums of complemented subspaces in locally convex spaces, Ark. Mat. 25 (1987), no. $1,147-153$.

Taras Shevchenko National University of Kyiv, Faculty of Mechanics and Mathematics, Kyiv, Ukraine

E-mail address: ivanmath007@gmail.com 